\title{
ESTRATEGIAS UTILIZADAS POR LAS EMPRESAS CONSTRUCTORAS EN SINCELEJO PARA TENER VENTAJAS COMPETITIVAS
}

\author{
STRATEGIES USED BY THE CONSTRUCTION COMPANIES IN COMPETITIVE \\ ADVANTAGES TO HAVE SINCELEJO
}

Alfredo Carlos Yemail Barragan* Corporación Universitaria del Caribe, CECAR

RECIBIDO: 10 AGOSTO DE 2013

ACEPTACIÓN: 10 OCTUBRE DE 2013

\section{RESUMEN}

El presente artículo es de revisión bibliográfica, tiene como objetivo mostrar las estrategias que utilizan las empresas de la construcción, en la ciudad de Sincelejo (Sucre, Colombia), para la generación de ventaja competitiva. Para ello, se consultaron, aproximadamente, 60 fuentes secundarias entre Journals, revistas científicas indexadas, y libros físicos y electrónicos de diferentes autores e investigaciones relativos a la temática planteada. Posteriormente, se realizó un análisis, obteniéndose como resultado las diferentes estrategias utilizadas por las empresas del sector de la construcción, para lograr ventaja competitiva.

Palabras clave: Estrategia, crecimiento, competitividad, clasificación JEL: M10, N66, O18.

\footnotetext{
*Administrador de Empresas, Especialista en Gerencia de Mercado, Gerente de Atlancic Constructora, correo: aymail@atlantisconstructora.com 


\begin{abstract}
This article of bibliographical review aimed to show the strategies used in the construction companies in the city of Sincelejo, (Sucre), Colombia, in order to gain competitive advantage. To achieve this purpose, 60 secondary sources of indexed scientific journals were consulted, as well as physical and electronic books of different authors and research projects with regard to the aforementioned subjectmatter. A further analysis was conducted obtaining as a result of this review, the different strategies used by the companies of the construction sector togain competitive advantage.
\end{abstract}

Key words: strategy, growth, competitiveness JEL Classification: M10, N66, O18 


\section{INTRODUCCIÓN}

El sector de la construcción es uno de los más importantes en el mundo, puesto que dinamiza la economía y el desarrollo de los países, al brindar, al mismo tiempo, oportunidades de empleo y bienestar a muchas familias gracias a la adquisición de viviendas y a la construcción de nuevos establecimientos comerciales e industriales. Ello se evidencia a través de numerosas investigaciones, que observan su desarrollo y las estrategias a las que apelan para obtener ventajas competitivas (Florez \& Rozo, 2012).

No obstante, al analizar los indicadores líderes sectoriales en Colombia, tales como los lanzamientos, iniciaciones, ventas y ofertas de vivienda VIS y 7 no VIS, se encuentra que, entre enero y octubre de 2012, el sector sufrió una caída promedio de más o menos $13,6 \%$. Además, los lanzamientos (-13\%), las ventas (-10\%) y las iniciaciones (18\%) presentaron, como se ve, una dinámica negativa, que se puede explicar por la "desaceleración en el consumo y los bajos 0 nulos estímulos a la demanda de vivienda durante la primera parte del año y por una reducción de la actividad en el distrito capital enmarcada por importantes restricciones a la oferta" (Ortega et al, 2012).

A pesar de lo anterior, es importante recordar que la base de comparación para el cálculo de los crecimientos anuales fue la del año 2011, el cual tuvo unos resultados extraordinarios al cierre, con crecimientos en: lanzamientos $(8,8 \%)$, ventas $(15,7 \%)$ e iniciaciones 
(20,6\%). Sin embargo, los niveles de iniciaciones estuvieron cerca de las 117.000 unidades, logrando alcanzar un pico histórico en esta actividad, por lo que sentó una base de comparación bastante alta con respecto al escenario 2012 (Ortega et al, 2012).

A pesar de lo dicho anteriormente, el comportamiento del sector edificador durante el primer semestre del 2013 resultó positivo. Según Salcedo et al (2013), en el primer trimestre del año, el valor agregado de este subsector creció $16 \%$ anual, aportando cerca del $50 \%$ al crecimiento total del sector constructor (edificaciones + obras civiles), cuyo crecimiento fue del $16,5 \%$ anual.

En este contexto, la región Caribe también presentó un crecimiento en el área aprobada para construcción, lo cual contribuyó significativamente sobre el desempeño nacional. En el caso de las licencias otorgadas para esta región, entre enero y marzo de 2013, se aprobaron 1.052.191 $\mathrm{m}^{2}$ para construcción, es decir, 391.477 $\mathrm{m}^{2}$ más que en el mismo periodo del año anterior, lo que constituye un aumento de 59,3\%. En concreto, se otorgaron permisos para la construcción, vivienda de interés social (VIS), vivienda diferente a VIS y bodega (Banco de la República, 2013).

En el caso específico del municipio de Sincelejo, en los últimos 5 años, el aumento de las actividades del sector de la construcción para sus diferentes usos (comercial, residencial e industrial) ha sido notorio, como se puede confirmar en 
el Informe de Banco de la República (2010). Este expresó que, durante el año 2010, se otorgaron en Sincelejo 192 licencias para construir, lo que frente al 2009 implica un aumento del $37,1 \%$, con un área de $143.392 \mathrm{~m}^{2} \mathrm{y}$ un incremento del 96,3\%, con respecto al año anterior, es decir, un crecimiento de $70.931 \mathrm{~m}^{2}$; mientras que en 2011, el mismo informe arrojó que el número de licencias de construcción aprobadas fue de 133, $30,7 \%$ menos que en 2010. Las licencias destinadas a la construcción de vivienda se situaron en $72,2 \%$ del total. En términos de área, se licenciaron $77.072 \mathrm{~m} 2$, con una caída de 66.320 m2 $(-46,3 \%)$ en comparación con el año anterior. Asimismo, la vivienda disminuyó en $37.303 \mathrm{~m} 2(-42,3 \%)$.
Para 2012, Sincelejo obtuvo 194 licencias de construcción, lo que constituye un aumento de 45,9\% frente a 2011; es decir, 61 licencias más. El área licenciada en sí alcanzó $129.500 \mathrm{~m} 2$, comprendiendo un aumento del $68,0 \%$ representado en $52.428 \mathrm{~m} 2$. En ese mismo año, a vivienda se le otorgaron 141 licencias, para un $41,0 \%$ del área por construir, o sea, $53.137 \quad \mathrm{~m} 2$ aprobados, lo que significa una variación de 4,6\% con respecto al año anterior (Banco de la RepublicaDANE, 2012).

Todas estas cifras sugieren la trascendencia que tiene el sector de la construcción en la vida económica de la región, ya que este contribuye a la creación de nuevos empleos, aumenta el crédito hipotecario y permite mejorar las condiciones de 
vida de sus habitantes, suscitando un efecto dinamizador en el resto de sectores económicos. A pesar de lo anterior, es evidente la carencia de información ordenada y sistemática, lo que dificulta su utilización como una herramienta legítima para conocer las condiciones reales del sector de la construcción en la ciudad de Sincelejo y su potencial en el futuro.

Ante esta situación, el presente estudio propone diferentes perspectivas y enfoques del sector objeto de investigación, para analizar las implicaciones que tiene específicamente en el municipio de Sincelejo. Al mismo tiempo, se trata de llevar a cabo un análisis estratégico de las constructoras ubicadas en la ciudad, con el objetivo de garantizar su permanencia en el mercado, tener mayor ventaja competitiva y establecer nuevas estrategias que permitan mejorar la competitividad y penetrar nuevos mercados, mejorando así su rentabilidad. De manera concreta, entonces, el presente artículo busca revisar los referentes teóricos e investigaciones que incluyan información valiosa sobre el sector de la construcción respecto a las diferentes estrategias que pueden ser utilizadas en él para lograr mayor competitividad y obtener ventajas competitivas.

\section{MÉTODO}

El método utilizado en la investigación es descriptivo con un enfoque cualitativo. En su desarrollo, se siguieron cuatro fases:

Fase 1. Revisión del estado del arte. Para perfeccionar el 
planteamiento de la pregunta de investigación, la cual orienta la realización del documento, se hizo necesario efectuar primero una amplia revisión en los aspectos conceptuales y métodos sobre el concepto de estrategia, crecimiento, competitividad y su medición.

Fase 2. Identificación de la pregunta de investigación. Con posterioridad a la fase de revisión, se precisaron la pregunta y el objetivo del documento.

Fase 3. Estudio de fuentes secundarias e identificación de los elementos de estrategia, crecimiento y competitividad en el sector de la construcción.

Fase 4. Propuesta de elementos con base en la metodología propuesta.

\section{APROXIMACIONES TEÓRICAS}

De la noción de estrategia a la ventaja competitiva

La estrategia es un elemento importante para las organizaciones porque ayuda a que estas sean competitivas y crezcan para enfrentar los desafíos del mercado. Por esta razón, a través de los tiempos, la estrategia se ha convertido en objeto de estudio de muchos investigadores y analistas, como se verá a continuación:

El estudio de la estrategia data de muchos siglos. Alrededor del año 500 A.C., el militar Sun Tzu afirmaba que el arte de la guerra consiste en someter al enemigo sin luchar $y$ hablaba en particular sobre la estrategia ofensiva, que se compara en la actualidad con los momentos en que una empresa quiere penetrar un 
mercado y considera la estrategia de resolver las dificultades antes de que se presenten, es decir, que antes de enfrentar el combate se debe vencer la estrategia del enemigo (Ballesteros, 2004).

Asimismo, Neumann y Morgenstern (1944, citados por Soto y Valente, 2005) analizan la llamada Teoría de los Juegos, en la cual la estrategia es una actividad en la que los participantes o jugadores llevan a cabo unas acciones y obtienen como consecuencia unos resultados que no sólo dependen de su propia acción sino de los otros participantes. Entre las limitaciones más características de este enfoque, aparece el hecho de considerar a los individuos como seres hiper-racionales que toman decisiones según un patrón matemático preestablecido.
Posteriormente, Chandler (1962) y

Andrews (1969, citados por Hoskisson et al, 1999) enmarcan estas primeras obras dentro de una perspectiva de contingencia (ajuste entre la estrategia y la estructura) y un marco basado en los recursos, enfatizando las fortalezas internas y debilidades. En este contexto, la estrategia se basa en la determinación conjunta de objetivos de la empresa y de las líneas de acción para alcanzarlas. El análisis de lo expuesto por los anteriores autores reconoce que la estrategia corresponde a una serie de planes que le permiten a la organización conseguir objetivos preestablecidos.

Las teorías sobre estrategia se movían en un plano poco cambiante, es decir, se basaban en situaciones totalmente previstas, dentro de las 
cuales se movían todas las decisiones de los directivos y que con seguridad terminarían en lo previsto, disminuyendo la incertidumbre en los resultados y generando confianza en las personas que llevaban a cabo la aplicación de estas estrategias.

En el enfoque de Mintzberg (2007), la estrategia de una organización se ha llevado a cabo en realidad como un patrón en una secuencia de acciones. Su investigación rastreó las estrategias

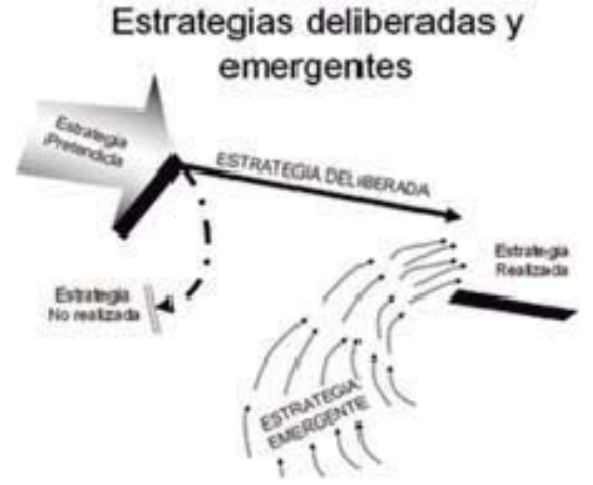

Fuente: Mintzberg (1987: 14).

Un enfoque importante para la planeación estratégica es el en las organizaciones durante largos períodos de tiempo, por lo general tres o cuatro décadas y, en un caso, hasta de un siglo y medio. Esto puso de manifiesto los patrones de las estrategias en la forma "deliberada"; el cambio en las organizaciones, con la interacción de las estrategias "emergentes", y las relaciones entre el liderazgo, la organización y el medio ambiente en el proceso de formación de la estrategia.

establecido por Porter (1980). Según este autor, existen cinco fuerzas que determinan las consecuencias de rentabilidad a largo plazo de un mercado o de algún segmento de éste. Por su parte, David (2003: 98) describe la estrategia competitiva como: "las acciones ofensivas 0 defensivas de una empresa para 
crear una posición defendible dentro de una industria", acciones que eran la respuesta a las cinco fuerzas competitivas que el autor indicó como determinantes de la naturaleza y el grado de competencia que rodeaba a una empresa y que buscaba obtener, como resultado, un importante rendimiento sobre la inversión.

Ahora bien, la competitividad, como concepto del management, se ha convertido en tema de análisis por parte del mundo académico y empresarial, al punto que las diferentes acepciones propuestas han permitido construir de manera consensuada una aproximación a lo que el mundo globalizado de hoy exige. Es así como, a partir de los diferentes significados del término competencia, se han de generar los espacios necesarios para entender la dimensión de competitividad (David, 2003:98).

De otra parte, la combinación de tierra, trabajo y capital se convierten en elementos indispensables para que surjan condiciones diferenciadoras entre países. En otras palabras, la combinación de recursos naturales, bajo costo en la mano de obra, etc., permiten que algunos países, en comparación con otros, sean más competitivos, ya que la producción de bienes y servicios se hace más barata, desplazando la competencia del mercado (Porter, 1980).

Cuando surgió el llamado pensamiento neoclásico, la conceptualización sobre competencia comenzó a ser analizada desde una 
perspectiva lineal, centrada debe tener una estructura más esencialmente en un modelo de pequeña comparada con el mercado, equilibrio general de competencia evitando así el control de precios por perfecta. Así, en el mercado libre, se parte de algunos productores (Aktouf, concibe la necesidad de una 2009). interferencia institucional para que exista un buen funcionamiento. Además, cada unidad económica Esta línea de argumentación coincide con lo que Porter (1980) propone en su modelo de competitividad estratégica, en el sentido de que toma acciones ofensivas o defensivas para crear una posición defendible en una industria, con la finalidad de afrontar con éxito a las fuerzas competitivas y generar un retorno sobre la inversión. Según el mismo Porter, el soporte sobre el que las empresas fundamentan un desempeño superior al promedio radica en la ventaja competitiva sostenible, desde el cual, las firmas

que compiten en el mercado junto a la participación del Estado, se convierten en una importante fuente generadora de recursos que favorecen el entorno y estabiliza las políticas macroeconómicas. Del mismo modo, Chiri (2011: 29) expresa que "la competitividad está determinada por la productividad con que un país usa sus recursos". En un sentido más amplio, se dice que la competitividad depende de la calidad $y$ los atributos de los bienes y servicios y de la eficiencia con la que son producidos. 
Ventaja Competitiva de las Naciones,

Por otra parte, la Comisión Económica para América Latina y el Caribe propone que la "competitividad es el proceso de expansión de la oferta exportable y penetración de mercados externos, con el consecuente mejoramiento en el nivel de vida de la población" (Villarreal, 2003: 187). Este enfoque cobra fuerza porque en el contexto actual las instituciones adquieren importancia en los mercados globales, además de generar espacios propicios para que los competidores interactúen de manera armonizada bajo unas normas establecidas.

Finalmente, en la década de los 80 se presenta la competencia desde una perspectiva conceptual nueva, a la que Porter (1980) denominó: que es vista desde un punto de vista macroeconómico. Ella implica el desarrollo de habilidades y de un desempeño particular en las empresas para que se logren los objetivos de manera permanente, puesto que la ventaja competitiva sostenida se logra cuando la empresa alcanza resultados destacados en varios años (Hill y Jones, 2005).

En conclusión, para la obtención de la ventaja competitiva, no existe una estrategia competitiva universal y sólo podrán alcanzar el éxito las estrategias adaptadas al sector y a las técnicas y activos de una empresa en particular (Porter, 1991: 64).

\section{Evolución y estrategias del} sector de la construcción 
La actividad de la construcción alude a todos los proyectos en los cuales se ejecutan obras tanto públicas como privadas, realizadas por empresas constructoras, contratistas o sub-contratistas, que casi siempre se relacionan con el sector industrial y todo lo que este ofrece en materia de servicios derivados. En este contexto, se produce una interrelación entre el sector de la construcción en la extensión de su cadena de valor y el sector manufacturero que produce los materiales y los proveedores. Al mismo tiempo, el sector ofrece una infraestructura que brinda auge y desarrollo, por lo que es considerado como uno de los más importantes en el ámbito local y global.

Concretamente, según el estudio realizado en España acerca de la competitividad de la industria de la construcción, "la estructura del sector así definido resulta heterogéneo y agrupa algunas actividades que se tratan como sectores diferenciados. Tal es el caso de la extracción de piedra, arena y arcilla, o de la fabricación de materiales para la construcción" (Observatorio Industrial del Sector de la Construcción-OISC, 2011).

En el contexto mundial, las investigaciones apuntan a diagnosticar el comportamiento y la evolución del sector de la construcción durante los últimos años, haciéndose notorio el auge del mismo según las proyecciones propuestas en el estudio realizado por Florez \& Rozo (2012:30): "en la actualidad la industria de la construcción es responsable 
aproximadamente del $10 \%$ del PIB mundial, genera alrededor del $7 \%$ de empleos y usa un 50\% de recursos naturales, mostrando que es uno de los sectores más importantes para el desarrollo de los países".

Por otra parte, en otro contexto se mantiene la perspectiva de la importancia y el dinamismo que genera a un país este sector, como es el caso de Chile. Así, citando a Porter et al (2007), el estudio realizado por la Cámara Chilena de la Construcción plantea: "La construcción incrementará su competitividad en la medida que realice acciones conducentes a elevar su productividad" (Corporación de Desarrollo Tecnológico-CDT, 2008: 58).
También Eyzaguirre, refiriéndose al caso Chile (2007:48), plantea que se requiere incrementar la productividad total de los factores, ya que el aporte de los factores de producción clásicos (capital y trabajo) muestra una tendencia decreciente. Al respecto, el mismo autor señala:

En el mundo existe hoy un amplio consenso en torno a que la respuesta para el crecimiento de largo plazo está en la evolución de la Productividad Total de Factores (PTF), y en Chile, durante buena parte de los últimos 20 años el aporte de la PTF ha sido importante. Esto puede constituir un problema a mediano plazo porque la posibilidad de crecer sostenidamente, sobre la base de capital y mano de obra no calificada, no dura para siempre. 
constructora debe utilizar información

Esto significa que entre las estrategias requeridas para lograr ventajas competitivas, la capacitación de la mano de obra ocupa un lugar importante. Como $\mathrm{Y}$ que es indispensable potencializar los recursos disponibles para mejorar la infraestructura en cada uno de los factores de la productividad.

La globalización también ha impactado significativamente a las empresas constructoras, debido a los efectos que rodean los entornos competitivos. Actualmente, los conocimientos de ingeniería, construcción y sus tecnologías son de dominio público, así como el uso de materiales, el empleo de técnicas, procedimientos y la manera como está organizada la inversión en I + D + i. Por esto, hoy día, una abierta y disponible para todos. Además, debe generar sus propias estrategias, basándose en su capital intelectual para crear ventajas competitivas específicas, a fin de optimizar la agregación de valor. Del mismo modo, la forma como se combina el capital intelectual y sus recursos es de vital importancia para la empresa (Morales \& Blanco, 2007).

Para el caso de Colombia, autores como Florez \& Rozo (2012: 35) explican que "el sector económico de la construcción juega un papel muy importante en el dinamismo de la economía nacional". Y, precisamente, por su alto nivel de encadenamiento con otros sectores productivos que al mismo tiempo actúan como un factor multiplicador de producción y empleo, se genera la necesidad de realizar 
investigaciones que potencialicen el crecimiento de las empresas del sector.

Por otro lado, la estabilidad económica que se observó en el país al finalizar la década de los años 90 propició una situación favorable para este sector, que se extendió hasta mediados del 2007. En este sentido, cuando se incrementaron los niveles de empleo, bajaron las tasas de inflación y, por ende, los servicios financieros ofrecieron tasas accesibles para los usuarios de los créditos hipotecarios (Galindo \& Mosquera, 2008). No obstante, el año 2008 marcó un antes y un después, dada la crisis económica internacional que afectó todos los sectores productivos, a lo que el sector de la construcción no fue ajeno. Esta crisis se mantuvo hasta el 2009, año durante el cual cayeron considerablemente en Colombia el crédito hipotecario y los indicadores en torno al sector de la construcción. Como medida contra cíclica, el Estado creó una cobertura condicionada al subsidio a la tasa de interés de los créditos hipotecarios, en proporción inversa al precio de las viviendas (Urrutia \& Namen, 2011).

La Cámara Colombiana de la Construcción-CAMACOL realiza constantemente estudios del sector y ha encontrado que una de las estrategias para la competitividad es el modelo de clusters. El Consejo Privado de Competitividad visionó este modelo, que parte de un esquema nuevo, en el cual hay iniciativas de colaboración entre compañías, entidades públicas, comunidad científica e instituciones 
financieras que manejan un grupo de actividades

económicas

interrelacionadas y que buscan

mejorar el desempeño en una región geográfica específica (Chirivi \& Pulido, 2009).

Aunado a lo anterior, las empresas constructoras han establecido estrategias de crecimiento que les han permitido mantenerse como uno de los sectores más importantes de la economía de un país. Entre estas se encuentran las de integración vertical, que tienen lugar cuando la función correspondiente a dos o más miembros del canal, situados a distinto nivel, es dirigida o administrada por alguno de sus componentes. El resultado de la integración vertical es una red de establecimientos, situados a distinto nivel del canal y dirigidos como un sistema de distribución centralizado (Santesmases, 1996: 467).

Se considera que una empresa está más integrada verticalmente cuando organiza por sí misma un mayor número de fases de un mismo proceso productivo. Esta integración puede ser hacia adelante o hacia atrás. La exploración de la ventaja competitiva, que busca dar explicación a la estructuración de los canales que suponen la eficiencia, se encuentra en Bucklin (1971) y en Bucklin et al (1996). Para este autor, la estructura del canal tiene dos grandes componentes: el sector comercial (el sector distribuidor o redes de intermediación) y el sector de consumo final.

Cruz (1990: 274) distingue tres tipos básicos de integración vertical: 
corporativas, contractuales y administradas. Todas ellas se refieren a la situación a distinto nivel de los miembros del canal. Así, mientras que en la primera uno de ellos tiene la propiedad del mismo; en la segunda, se asignan funciones y actividades mediante la negociación, el diseño y la ejecución de un contrato que vincula en su totalidad los canales de distribución o a una parte; y en la tercera, los distintos miembros de un canal de distribución ejercen el control y desarrollo sin que existan relaciones de propiedad o vinculación contractual.

La integración vertical, sea corporativa, contractual o administrada, se puede producir en dos direcciones: hacia arriba o hacia atrás y hacia abajo o hacia adelante. La primera se presenta cuando la empresa minorista integra en su organización actividades mayoristas e incluso manufactureras, o cuando una organización mayorista introduce actividades de fabricación. La segunda se produce siempre que una empresa manufacturera realiza funciones de mayorista $y / o$ de minorista, o cuando un mayorista realiza a la vez funciones de minorista con el fin de ser más eficientes y ejercer mayor control. (Vázquez y Trespalacios, 1997, citado por Cuesta, 2006).

"Los beneficios que reporta la integración dependen de la capacidad productiva de las empresas y de la competitividad de cada una de ellas" (Montoya, Montoya, \& Castellanos, 2010: 114). De hecho, si esto no se cumpliera, resultaría necesario comprar o vender en el mercado 
abierto con el fin de minimizar los costos que pueda ocasionar un efecto contrario, es decir, desventajas competitivas.

Para el proceso de integración vertical en situaciones de competencia, lo anterior trae como consecuencia que la elección entre un canal directo (integrado) o un canal indirecto (no integrado) se terminará cimentando en la eficiencia relativa de cada uno de ellos, de manera que pueda proveer el nivel de servicios de distribución reclamados por el usuario final.

Mediante la organización vertical de los canales de distribución, los participantes en las organizaciones buscan conseguir tanto la consecución de un mayor poder de mercado (hacia la demanda), como un mayor poder de negociación (hacia los proveedores) (Casares y Rebollo, 1996:109)

$Y$ es que, en el sector de la construcción, ocurre a menudo que la demanda y la cadena de suministro no están bien integradas (Vrijhoef y Ridder, 2005, citados en Carbonel, 2011). Esto se considera lógico, debido a que en la práctica la integración de los sistemas debe ser costosa y compleja, sobre todo en una industria que debe organizarse principalmente por medio de proyectos. Al respecto, Vrijhoef (2007, citado en Carbonel, 2011) propone dos sistemas: un sistema integrador de la demanda y un sistema integrador de la cadena de suministro en este sector.

En este sentido, cuando se piensa en establecer una estrategia de crecimiento, se pueden identificar 
algunos problemas de tipo cualitativo, que están relacionados con la necesidad de cambiar la estructura, y los métodos de dirección y de control; pero también aparecen problemas cuantitativos, que se desprenden de las demandas de los recursos para la inversión y financiación que ese crecimiento genera (Salas Fumás, 1986). Autores como Correa, González y Acosta (2001) aseveran que el crecimiento empresarial no cuenta con una teoría general ni mucho menos con una definición conceptual consensuada porque los conceptos sobre este se basan en diversos enfoques sobre la extensión, los parámetros de medida y algunos factores determinantes.

No obstante, la planteada por Blazquez (2005) se considera como una definición bastante acertada.
Según este, el crecimiento empresarial es:

El proceso de adaptación a los cambios exigidos por el entorno o promovido por el espíritu emprendedor del directivo, según el cual, la empresa se ve compelida a desarrollar o ampliar su capacidad productiva mediante el ajuste o adquisición de nuevos recursos, realizando para ello cambios organizacionales que soporten las modificaciones realizadas. (P. 16)

Basado en lo anterior, y teniendo en cuenta que el crecimiento empresarial abarca procesos de integración empresarial, desde una aproximación basada en los resultados, se favorecen las posibilidades de las organizaciones que se integran. Según Garzón 
(2005), entre otros beneficios que

aporta, esto posibilita el reforzamiento

de competencias, genera ventajas vía

integración vertical y horizontal y,

además, produce otras ventajas en

eficiencia de estrategias coordinadas

entre agentes.

Es importante resaltar que las estrategias de crecimiento de las empresas en las que el resultado esperado es la disminución de los costos se sustenta en la teoría de los costes de transacción, los cuales son aquellos en los que se incurre al planificar, adaptar y supervisar la realización de las tareas bajo las estructuras de gobierno alternativas (Williamson, 1985).

Algunos de los desarrollos más recientes que utilizan esta teoría se enfocan en las estrategias de outsourcing y, concretamente, en la toma de decisiones vinculadas con el establecimiento del proceso (López et al, 2002; Caniels y Roeleveld, 2009; Chen, 2009; Lamminmaki, 2009; Bhagat et al, 2010 y Schoenherr, 2010) y en la integración vertical (Joskow, 2010; Bucheli et al, 2010)

Velásquez (2004) plantea que en la integración se hace indispensable la concertación de voluntades. Además, se deben establecer contratos formales de compromiso que conlleven a la posibilidad de constituir nuevas formas jurídicas y, de esta manera, al establecimiento de contratos formales de compromiso, y a la posibilidad de constituir nuevas formas jurídicas e impedir la competencia desleal y el oportunismo en el ejercicio de la misma.

En ese orden, uno de los procesos involucrados es la gestión 
de la cadena de suministro. De acuerdo con Stanley \& Stanley (1995), los principios para la gestión de la cadena de suministro consisten en gestionar los recursos de las empresas implicadas con una visión sistémica de los flujos y procesos de negocio, con el objetivo de lograr una calidad de servicio hacia los clientes, minimizando el coste total de la cadena de suministros. Al respecto, Jagdev \& Browne (1998) afirman que los productores responden a los cambios competitivos, trabajando de manera integrada con sus proveedores y clientes y construyendo una empresa extendida a través de la cadena de valor. Esto evidencia que debe existir una relación entre empresas independientes en cuanto a la coordinación en el diseño, desarrollo
Al hacer un análisis de las empresas del sector, se nota que manejan muchos proyectos en diferentes zonas geográficas al mismo tiempo y que, a su vez, tienen invertidos grandes capitales en dichos proyectos (Rey \& Echeverry, 2005).

Para mejorar la capacidad competitiva y lograr una apropiada sinergia, se necesita una buena estrategia como base del crecimiento organizado y estructurado, en la que convergen las diversas estrategias, cuyo único fin es garantizar la perdurabilidad de las organizaciones en el tiempo, siendo reconocidas y generadoras de valor.

\section{Análisis del contexto} competitivo: caso constructoras de

\section{Sincelejo}

y costes. 
En el municipio de Sincelejo, existen alrededor de 25 empresas pertenecientes al sector de la construcción, que construyeron apartamentos o conjuntos cerrados para los estratos 4,5 y 6 . La rivalidad entre los competidores es grande, debido a la pequeña cantidad de proyectos de este tipo que se presentan en la ciudad. Esto se demuestra en la expedición de apenas 40 licencias de construcción para proyectos de este tipo por parte de las curadurías urbanas N1 y N2 de Sincelejo, lo que nos da un promedio de 4 proyectos de vivienda al año.

En este grupo de 25 constructoras, hay cuatro que lideran el mercado de la construcción de apartamentos o conjuntos cerrados mayores a 6 unidades habitacionales en los estratos 4, 5 y 6 . La empresa que encabeza la lista es Atlantis Constructora Ltda., seguida por Promotora Villas del Mediterráneo Ltda. En tercer lugar, encontramos a Isaac y Durán Ltda. y, por último, a Dorian Lastre, Construelite.

Las empresas constructoras que pretendan entrar al mercado local deben considerar el liderazgo de costos que poseen las empresas establecidas en la ciudad, los cuales les permiten ofrecer sus proyectos a menores precios debido a que sus costos de producción son más bajos.

Una de las estrategias utilizadas en este contexto ha sido la forma de obtener beneficios por la negociación con los proveedores en cuanto a la cantidad de los materiales para la construcción, puesto que comprando grandes cantidades de éstos se consiguen descuentos que 
disminuyen los costos y esto permite ofrecer precios más competitivos. Actualmente, el sector de la construcción se caracteriza por ser de una naturaleza cambiante y constantemente se enfrenta a nuevos retos, por lo que las empresas deben estar preparadas para enfrentarlos (Pereira \& Chaparro, 2004).

Un factor de influencia importante para el sector constructor en Sincelejo fue la pasada ola invernal, causada por el fenómeno de La Niña, que vivió el país durante los años 2010 y 2011. Este fenómeno generó graves daños de infraestructura y vivienda, no solo en el municipio de Sincelejo sino también en varios municipios del departamento de Sucre.

Los hechos anteriores generan una oportunidad muy importante para el sector de la construcción, puesto que Colombia Humanitaria presupuestó 139 mil 575 millones de pesos, que deben utilizarse para ejecutar 383 obras de rehabilitación y para la atención humanitaria en el departamento de Sucre. Las obras que contempla este presupuesto están distribuidas en varios programas para la superación de la etapa de emergencia.

Finalmente, un factor que afecta negativamente al sector constructor es el aumento del precio del cemento gris y acero, ya que este constituye uno de los insumos de mayor importancia para este sector. La empresa Cementos Argos aumento en un $4 \%$ el precio del cemento gris, lo que equivale a $\$ 1.000$ más por bolsa. Ello no solo afecta el presupuesto de construcción de la 
obra en este elemento específico, sino también el precio de otros insumos, como los bloques, generando un mayor costo de construcción, pero además perjudica al comprador del inmueble, puesto que estos costos son transferidos a él al aumentar el precio final de la vivienda.

\section{CONCLUSIÓN}

El presente documento se inició resaltando la idea de estrategia, crecimiento y competitividad en el sector de la construcción, como los elementos fundamentales para la generación de valor en el desarrollo de la actividad edificadora, tanto para las organizaciones en particular como para el sector. diferentes postulados, existe un consenso respecto a que no hay estrategia perfecta. En cambio, lo que sí se encuentra en la bibliografía es que este es un concepto integral que involucra a las empresas a nivel micro y macro.

Conocer el comportamiento del pasado y del presente en el sector de la actividad edificadora y los cambios de tendencia, resulta fundamental para entender y proponer estrategias necesarias en el futuro cercano de uno de los sectores más importantes de la economía en Colombia.

Este documento se presenta como una herramienta de consulta y análisis desde el enfoque académico, pero se advierte sobre la dificultad para acceder a la información y los escasos referentes sobre el tema.

Como se manifiesta en el cuerpo de los artículos y en los 
REFERENCIAS

Aktouf, O. (2009). La Administración entre Tradicion y Renovación. 4a edicion. en español. Cali: Universidad del Valle, Gaëtan Morin Éditeur.

Ballesteros, M. (2004). Fundamentos de la estrategia para el Siglo XXI. Ministerio de Defensa Nacional, $15-64$.

Banco de la Republica-DANE. (2012). Informe de Coyuntura Economica Regional - Sucre. Banco de la Republica -

DANE.

Banco de la República. (2010). Informe de Coyuntura Económica Regional . Sucre: Banco de la Republica-DANE.

Banco de la República. (2013). Panórama Económico
Regional. Boletin Economico Regional, 1 - 46.

Bhagat, P., Byramjee, F., \& Taiani, V. (2010). A Framework of Total Value Orientation for Strategic Outsourcing Decisions. Competitiveneses Review, Volumen 20, Número 4, 305.

Blazquez, F. (2005). Los Incentivos como Factor del Crecimiento Empresarial: Análisis Empírico de la Reserva para Inversiones en Canarias. Tesis de Doctorado. España: Universidad de Las Palmas de Gran Canaria.

Bucheli, M., Mahoney, J., \& Vaaler, P. (2010). Chandler's Living History: The Visible Hand of Vertical Integration in Nineteenth Century America Viewed Under a Twenty-Firat 
Century Transaction Cost

Decisions.

European

Economics Lens. The Journal

Management Jorunal, Vol. 27

of Management Studies,

Número 6, 402.

Volumen 47, Número 5, 859.

Bucklin, L. (1971). Vertical Marketing

Carbonel, F. (2011). Modelos de Systems. Scott Foresman and Company.

Bucklin, L., Ramaswamy, V., \& Integracion de la Gestion de la Cadena de Suministro. Direccion y Organizacion No. 43, Valencia .

Majumdar, S. (1996).

Chen, S. (2009). Transaction Cost Analyzing Channel Structures Rationale for Private Branding of Business Markets via The and its Implications for the Structure Output Paradigm. Choice of Domestic vs International Journal of Offshore Outsourcing. Research in Marketing Vol. 13, Volumen 40, Número 1, 156 $73-87$. 176.

Caceres, R., \& Rebollo, A. (1996). Distribucion Comercial. Madrid:

Chiri, A. (2011). Marco Analítico de la Civitas.

Caniels, M., \& Roeleveld, A. (2009). Power and Dependence Competitividad de Michael E. Porter: De la Teoría a la Práctica. Perú: Editorial Perspectives on Outsourcing 
Chirivi, E., \& Pulido, L. (2009). Cuesta, P. (2006). Estrategias de Lecciones del Congreso Crecimiento de las Empresas Colombiano de la Construcción 2009:. Estudios Economicos CAMACOL, 1 - 8. de Distribucion Comercial. España:

http://www.eumed.net/tesis/20

Corporacion de Desarrollo Tecnologico. (2008). Estudio 06/pcv/. consultado agosto de 2013.

de Competitividad del Sector Construccion en Chile: marzo abril de 2008. Chile:

Corporacion de Desarrollo Tecnologico.

Correa, A., Gonzalez, A., \& Acosta, M. (2001). Crecimiento el la Pyme [sic.] Canaria: Influencia del Tamaño, la Edad y el Sector de Actividad. Universidad de la Laguna. Documento de trabajo.

Cruz, I. (1990). Fundamentos de Marketing. Barcelona : Ariel.
David, F. (2003). Conceptos de AdministraciónEstratégica. Mexico: Pearson Prentice Hall.

Eyzaguirre, N. (2007). Conocimiento e Innovacion para el Crecimiento. En P. Cortez, Emprendimiento e Innovacion en Chile (págs. 39 - 80). Chile: Universidad del Desarrollo.

Florez, A., \& Rozo, D. (2012). Thanatos Empresarial: Evolución del Sector de la Construcción en Colombia. 
Trabajo de Grado. Bogota

D.C.: Universidad del Rosario.

Galindo, J., \& Mosquera, M. (2008).

Notas del Mercado de

Capitales: Construcción en

Colombia:

Riesgos

elncidencias en el sector

financiero. BRC INVESTOR

SERVICES S.A .

Garzon, D. (2005). Programa sobre Operaciones Colectivas entre

Empresas. Seminario

Iberoamericano sobre

Integracion Empresarial y

Cooperativa: cooperar y

exportar para Ganar. Convenio

BID-ATN7ME 7856 CO,

www.iberpymeonline.org/venez

uela130705/ricargogarzon.pdf

consultado agosto de 2013.

Hill, W., \& Jones, G. (2005).

Administración estratégica, Un enfoque Integrado $6^{\circ}$ Edicion .

Mexico: McGraw Hill.
Hoskisson, R., Hitt, M., \& Yiu, D. (1999). Theory and Research in Strategic Management: Swings of Pendulum. Journal Management University of Oklahoma, 417 - 456.

Jagdev, H., \& Browne, J. (1998). The Extended Enterprise a Context for Manufacturing. Production Planning Control Vol. 9 No. 3, $216-229$.

Joskow, P. (2010). Vertical Integration. Antirust Bulletin, volumen 55, número 3, 545 587.

Lamminmaki, D. (2009). An Investigation of the Role Played by Frequency and Uncertainty in Hotel 
Outsourcing Decisions. Oxford . Oxford: Oxford Inernational Journal of University Press.

Services Technology and Management Vol. 11 Número 2, 182.

Montoya, A., Montoya, I., \& Castellanos, O. (2010). Situacion de la Competitividad Lopez, S., Ventura, J., \& Gonzalez, de las Pyme en Colombia: M. (2002). La Formalización de los Acuerdos de Elementos Actuales y Retos. Agronomia Colombiana, 107 Subcontratación: El Caso de la 117.

Industria Electrónica Española. Investigaciones Económicas Vol. 26 Número 1, 87 -111.

Mintzberg, H. (1987) The Strategy aportación para el Futuro. Concept I: Five Ps For Facultad de Ciencias Strategy. California Económicas: Investigación y Management Review; Fall Reflexion. Rev.fac.cienc.econ. 1987; 30, $1 ; \quad$ ABI/INFORM [online], vol.17, n.2 [cited 2013Global pg. 11

10-09] Available from: $<h t t p: / / w w w . s c i e l o . o r g . c o / s c i e l$

Mintzberg, H. (2007). Tracking Strategies: Toward a General o.php?script=sci_arttext\&pid=S

Theory of strategy formation 
$68052009000200003 \& / n g=e n \&$

$n r m=i s o, 23-44$.

Morales, M., \& Blanco, A. (2007). La

Competitividad en la Industria de la Construcción en Mexico. UAM-AZC Anuario, 51 - 61.

Observatorio Industrial del Sector de la Construcción - OISC. (2011). La competitividad de la industria de la construcción en España. España: Observatorio Industrial del Sector de la Construcción - OISC.

Ortega, K., Quiroz, O., Sarmiento, V., \& Torres, J. (2012). Lo que se Avecina para la Actividad Edificadora. Estudios Económicos CAMACOL, 1 12.

Pereira, A., \& Chaparro, J. (2004). Un Sistema de Información
Interorganizacional

como

Soporte a la Gestión en el Sector de la Edificación. ACEDE.

Porter, M. (1980). Competitive Strategy. New York: Free Press 450p.

Porter, M. (1991). La Ventaja Competitiva de las Naciones. Argentina: Vergara.

Porter, M., Ketels, C., \& Delgado, M. (2007). The Microeconomic Foundations of Property: Finding from the Business Competitivenesss. World Economic Forum.

Rey, A., \& Echeverry, D. (2005). Prototipo para el Control de Obras y Teorías para el Manejo de Excesos de Liquedez. ICYA 2001-060. 
Ricardo, D. (1973). Principios de Economía Política y Tributación. Mexico: FCE.

Salas Fumás, v. (1986). ¿Conoce el Crecimiento Sostenible por su Empresa? Alta Dirección, 185 193.

Salcedo, M., Ortega, K., Sarmiento, V., Rueda, C., \& Silva, G. (2013). BALANCE DE LA ACTIVIDAD EDIFICADORA: I SEMESTRE DE 2013. Estudios Económicos CAMACOL, 1 - 13.

Santesmases, M. (1996). Terminos de Marketing: Diccionario Base de Datos. Mexico: Ediciones Pirámide S.A.

Santos, J. (2011). La Empresa Constructora en su Desenvolvimiento Jurídico.
Especial Atención a la Contratación con las Administraciones Públicas. Tesis doctoral. Guadalupe: Universidad Católica San Antonio.

Schoenherr, T. (2010). Outsourcing Decisions in global Supply Chains: An Exploratory Multi.country Survey. International Journal of Production Research, Vol. 48, número 2, 343.

Soto, A., \& Valente, M. (2005). Teoría de Juegos; Vigencia y Limitaciones. Revista de Ciencias Sociales (on line) http:/www.scielo.org.ve/scielo. php?scipt=sci_arttext\&pid=S13 $15-$ 95182005000300008\&ing=es\& nrm=iso, $497-506$. 
Stanley, E., \& Stanley, A. (1995). The

Firm as a Value-Added

System; Integrating Logistics,

Operations and Purchasing.

International Journal of

Physical Distribution \&

Logistics Management Vol.25

No. 5, 24 - 42.

Urrutia, M., \& Namen, O. (2011).

Historia del Crédito Hipotecario

en Colombia. Documentos

CEDE.

Vasquez, R., \& Trespalacios, J.

(1997). Distribucion Comercial.

Estrategias de Fabricantes y

Detallistas. Madrid: Civitas.

Velasquez, F. (2004). La Estrategia, la Estructura y las Formas de Asociación: Fuentes de Ventaja Competitiva para las

Pymes Colombianas. Estudios

Gerenciales , 73 - 97.
Villarreal,

R. (2003).

$\mathrm{La}$ Competitividad Sistemica: Conceptos y Condiciones en México. Perspectivas y Retos de la Competitividad en Mexico (págs. 187 - 208). Mexico: UNAM.

Vrijhoef, R. (2007). Supply Chain Integration in Construction. Design and Construction Processes. Delft Technische Universitiet Delft. Paper Series 2.

Vrijhoef, R., \& De Ridder, H. (2005).

Supply Chain Integration for Achieving Best Value for Construction Clients: Client Driven versus Supplierdriven Integration. Brisbane: Proceedings QUT Research Week. 
Williamson, O. E. (1985): The

Economic Institutions of

Capitalism. Firms, Markets,

Relational Contracting, The

Free Press, Nueva York 